\title{
Reduction of 2,3-bis(2-methoxy-4-nitro-5- sulfophenyl)-2H-tetrazolium-5-carboxanilide inner salt (XTT) is dependent on CaFRE10 ferric reductase for Candida albicans grown in unbuffered media
}

Correspondence

Simon A. B. Knight

sknight@mail.med.upenn.edu

Received 13 January 2006

Revised 28 March 2006

Accepted 12 April 2006

\section{Simon A. B. Knight and Andrew Dancis}

\author{
University of Pennsylvania School of Medicine, Department of Medicine, Division of \\ Hematology/Oncology, 730 BRB II/III, 421 Curie Boulevard, Philadelphia, PA 19104-6160, \\ USA
}

\begin{abstract}
The reduction of 2,3-bis(2-methoxy-4-nitro-5-sulfophenyl)-2H-tetrazolium-5-carboxanilide inner salt (XTT) and other tetrazolium salts is widely used as an assay for bacterial, fungal and mammalian cell viability, but the genes encoding the reductase activities have not been defined. Here, it was shown that XTT and plasma membrane ferric reductase activities were 10-40-fold greater in Candida albicans than in Saccharomyces cerevisiae. XTT reductase activity was induced fivefold in C. albicans grown in low-iron conditions compared with iron-replete conditions, and for cells grown in unbuffered $(\mathrm{pH} \mathrm{4.0-4 \cdot 4)} \mathrm{medium,} \mathrm{XTT} \mathrm{reductase} \mathrm{activity} \mathrm{was} \mathrm{largely} \mathrm{dependent} \mathrm{on}$ CaFRE10. XTT reductase activity of $C$. albicans grown in medium buffered to $\mathrm{pH} 6 \cdot 8$ was independent of $\mathrm{CaFRE} 10$ but, nonetheless, was upregulated in cells deprived of iron. Reduction of 2-(4,5-dimethyl-2-thiazolyl)-3,5-diphenyl-2H-tetrazolium bromide (MTT), a membrane-permeable tetrazolium salt, occurred at an intracellular location and was independent of CaFRE10. However, MTT activity was induced by iron deprivation in C. albicans but not in S. cerevisiae. C. albicans possessed multiple iron- and $\mathrm{pH}$-regulated reductase activities capable of reducing tetrazolium salts, but, when grown in unbuffered medium, CaFRE10 was required for XTT reductase activity.
\end{abstract}

\section{INTRODUCTION}

Tetrazolium salts are widely used as indicators of cellular proliferation and biomass for eukaryotic and prokaryotic cells (Johnsen et al., 2002; McCluskey et al., 2005; Roehm et al., 1991; Scudiero et al., 1988). Live cells reduce the tetrazole ring and a coloured formazan product is formed, which can be assessed visually and quantified spectrophotometrically. Such assays are used to test the efficiency of antifungal drugs in killing or inhibiting growth of Candida albicans and other fungi (Heyn et al., 2005; Kuhn et al., 2003; Meletiadis et al., 2001; Tellier et al., 1992; Wiederhold et al., 2005). The tetrazolium salts used in biology are aromatic derivatives of 1,2,3,4-tetrazole and include monotetrazolium [e.g. 2,3-bis(2-methoxy-4-nitro-5-sulfophenyl)-2H-tetrazolium-5carboxanilide inner salt (XTT), 2-(4,5-dimethyl-2-thiazolyl)-

Abbreviations: 1-mPMS, 1-methoxy-5-methylphenazinium, methylsulfate; BPS, bathophenanthroline disulfonate; FRE, ferric reductase; mtDNA, mitochondrial DNA; MTT, 2-(4,5-dimethyl-2-thiazolyl)-3,5diphenyl-2H-tetrazolium bromide; $\mathrm{NOX}, \mathrm{NAD}(\mathrm{P}) \mathrm{H}$ oxidase; SOD, superoxide dismutase; WST-1, 2-(4-iodophenyl)-3-(4-nitrophenyl)-5(2,4-disulfophenyl)-2H-tetrazolium monosodium salt; XTT, sodium 2,3bis(2-methoxy-4-nitro-5-sulfophenyl)-2H-tetrazolium-5-carboxanilide inner salt.
3,5-diphenyl-2H-tetrazolium bromide (MTT) and 2-(4iodophenyl)-3-(4-nitrophenyl)-5-(2,4-disulfophenyl)-2Htetrazolium monosodium salt (WST-1)] or ditetrazolium [e.g. nitro blue tetrazolium (NBT)] variants (reviewed by Berridge et al., 2005). XTT and WST-1 are reduced to form soluble coloured formazan products (Ishiyama et al., 1993; Paull et al., 1988; Peskin \& Winterbourn, 2000), and MTT, which can cross cellular membranes, forms water-insoluble formazan crystals upon reduction (Berridge et al., 2005; Mosmann, 1983).

Despite widespread use of these assays, the gene(s) and encoded enzymes that catalyse the reduction of the tetrazolium salts are still not known. Reduced pyridine nucleotides are likely to be the originating electron donors, but the specific reductases involved remain to be identified (Berridge \& Tan, 1993; Vistica et al., 1991). In mammalian cells, the plasma membrane is thought to be the site for the extracellular reduction of the soluble tetrazolium salts (e.g. XTT and WST-1), in a process that might involve NADH coenzyme Q-dependent ferricyanide reductases and a ubiquinone-dependent plasma membrane electron transport chain (Berridge \& Tan, 2000; Crane et al., 1985; Kim et al., 2002; Morre, 2004; Villalba et al., 1995). 
Whole cells of Saccharomyces cerevisiae can also reduce ferricyanide (Crane et al., 1982), and externally directed ferric reductases (FREs), encoded by the genes ScFRE1 and ScFRE2, are responsible for this process (Dancis et al., 1992; Georgatsou \& Alexandraki, 1994). The FREs have wide phylogenic distribution (Lara-Ortiz et al., 2003), and, in C. albicans, there are at least 11 FRE family members (Arnaud et al., 2005; http://www.candidagenome.org/). The FREs are, in turn, related to the large $\mathrm{NAD}(\mathrm{P}) \mathrm{H}$ oxidase (NOX) protein family. The archetypical NOX is gp91phox, which is present in human phagocytes, and responsible for superoxide production within the phagocytic vacuole (reviewed by Segal, 2005). Key features of NOX proteins that are conserved in FRE proteins include four conserved histidine residues for coordination of two $b$-haem groups within an intramembraneous region, and amino acid motifs associated with FAD and NADPH binding (Finegold et al., 1996). This configuration of cofactors permits the energetically favourable transfer of intracellular electrons from intracellular NADPH across the plasma membrane to an extracellular electron acceptor (Vignais, 2002).

In S. cerevisiae and C. albicans, a primary biological role of the FREs is the reduction of ferric iron chelates, thereby releasing ferrous iron and making it available to the highaffinity iron permease system, which consists of a multicopper oxidase (FET) and an iron permease (FTR) (Askwith et al., 1994; Eck et al., 1999; Knight et al., 2002; Kosman, 2003; Ramanan \& Wang, 2000; Stearman et al., 1996). This cellular iron-uptake system is critical for growth in ironlimiting conditions, and is homeostatically upregulated at the level of transcription in response to low levels of environmental iron (Blaiseau et al., 2001; Braun et al., 2000; Courel et al., 2005; Knight et al., 2002; Lan et al., 2004; Rutherford et al., 2005; Yamaguchi-Iwai et al., 1996). In studying the reductive iron-uptake system, we observed that C. albicans was able to reduce ferric and take up ferrous iron at a 40-100-fold greater rate than that of S. cerevisiae (Knight et al., 2005). ScFrelp is known to have broad substrate specificity, and can utilize a variety of electron acceptors as substrates, including nitroprusside, cupric chelates, resazurin and azo dyes, as well as ferric chelates (Hassett \& Kosman, 1995; Lesuisse \& Labbe, 1994; Ramalho et al., 2005). This led us to investigate if CaFre10p, the predominant externally directed ferric reductase of C. albicans when grown in typical laboratory conditions (Knight et al., 2005), was responsible for the reduction of tetrazolium salts used in the viability/biomass assays of C. albicans.

\section{METHODS}

Strains. C. albicans SC5314 was used as the wild-type reference strain. Other wild-type strains examined were CBS5736 (ATCC 32032) and a high-frequency switching strain, WO-1, kindly provided by David Soll, University of Iowa (Slutsky et al., 1987). Strain BCa15-2 harbours a homozygous deletion of CaFRE10 (also known as RBT2, CFL95) (Cafre10D: : hisG/fre10: : hisG-URA3hisG ura3 $\Delta:: i m m 434 /$ ura $3 \Delta:: i m m 434)$ and was a gift from Burk Braun and Alexander Johnson, University of California, San

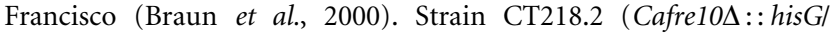
Cafre10:: hisG:: CaFRE10-URA3 ura3A:: imm434/ura3s:: imm434) harbours a reinserted functional CaFRE10 and CT212.2

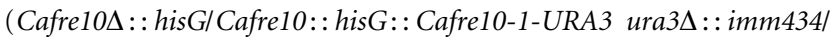
ura3s::imm434) harbours a reinserted mutated Cafre10 allele, and is auxotrophically matched to CT218.2 (Knight et al., 2005).

S. cerevisiae strains used were YPH499 [MATa ura3-52 lys2-801(amber)

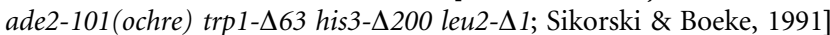
and the congenic ferric reductase knock-out 499 $1 \Delta 2$ [MATa ura3-52 lys2-801(amber) ade2-101(ochre) trp1- $663 \Delta$ fre1 : : LEU2 $\Delta$ fre2:: HIS3; Finegold et al., 1996]. Strain YPH250 rho is congenic with YPH499 (except for the presence of a $\operatorname{trp1-\Delta 1}$ allele) and was treated with ethidium bromide to ablate mitochondrial DNA (mtDNA) (Fox et al., 1991).

Growth conditions and media. For all experiments, strains were streaked from $-80{ }^{\circ} \mathrm{C}$ glycerol stocks onto $1 \%(\mathrm{w} / \mathrm{v})$ yeast extract, $2 \%(\mathrm{w} / \mathrm{v})$ peptone, $2 \%(\mathrm{w} / \mathrm{v})$ D-glucose and $0.01 \%(\mathrm{w} / \mathrm{v})$ adenine (YAPD) agar medium, grown for 2 days at $30^{\circ} \mathrm{C}$, then transferred to room temperature where they were maintained for a maximum of 10 days. All experiments were initiated from inoculation of a single colony to a single culture. For biological replicates, multiple single colonies were individually inoculated into multiple cultures. Defined iron-deficient medium was prepared from complete supplement mixture (CSM, $0.79 \mathrm{~g} \mathrm{l}^{-1}$; Q-BioGene) supplemented with $0 \cdot 1 \mathrm{~g}$ uridine $1^{-1}$, yeast nitrogen base (YNB, $6 \cdot 7 \mathrm{~g} \mathrm{l}^{-1}$, USBiologicals) without amino acids, iron, zinc, manganese or copper, and $2 \%(\mathrm{w} / \mathrm{v})$ D-glucose. This was supplemented with $0.4 \mathrm{mg}$ zinc sulfate $1^{-1}, 0.4 \mathrm{mg}$ manganese sulfate $1^{-1}$ and $1 \mu \mathrm{M}$ copper sulfate. The $\mathrm{pH}$ of this unbuffered medium was $4 \cdot 0-4 \cdot 4$. Milli-Q (Millipore) water was used for all growth media and chemical solutions. All iron-deficient media were prepared in plastic containers and filter-sterilized, and cells were grown in polypropylene or polystyrene vessels. The predicted iron concentration of this low-iron medium was of the order 50-100 nM (Eide et al., 1992).

Ferric, XTT, WST-1, and MTT reductase assays. The protocol for studying the reductase activities could be divided into three distinct stages: stage 1, growth of the cells in the specific medium; stage 2 , washing the cells in the assay buffer to remove the growth media; and stage 3, the enzyme assay. Using this protocol, we were able to compare the effects of the growth conditions on the reductase activities under identical enzyme assay conditions.

For all assays, cells were grown in 96-well plates with $150 \mu \mathrm{l}$ medium at $30{ }^{\circ} \mathrm{C}$ without shaking. After $17 \mathrm{~h}$, cells were resuspended and subinoculated $(1: 20)$ into fresh medium. The freshly inoculated cultures were incubated at $30^{\circ} \mathrm{C}$ for $5-6 \mathrm{~h}$. Cells were pelleted by centrifugation $(1000 \mathrm{~g}, 5 \mathrm{~min})$ at room temperature and washed twice with standard reductase buffer $[50 \mathrm{mM}$ citrate, $\mathrm{pH} 6 \cdot 6$, with $5 \%(\mathrm{w} / \mathrm{v})$ D-glucose]. $\mathrm{OD}_{720}$ of the cell pellets resuspended in reductase buffer was measured for cell number normalization, and assays were initiated by the addition of appropriate substrate (ferric ammonium sulfate, XTT, WST-1 or MTT; see details below) in reductase buffer to the cell suspensions. Reactions were carried out at $30{ }^{\circ} \mathrm{C}$ in the dark. After 10-15 $\mathrm{min}$, the ferric, XTT and WST-1 reactions were stopped by the addition of $25 \%$ trichloroacetic acid. We have previously determined that the reduction of ferric iron is linear over this time period (Knight et al., 2005). The cells were pelleted by centrifugation (1000 $g$ for $5 \mathrm{~min}$ ) and the supernatants were transferred to fresh 96-well plates for absorbance measurements (BioTek EL800 plate reader). The MTT reactions were stopped after $1.75 \mathrm{~h}$ with acidified $(0 \cdot 04 \mathrm{M} \mathrm{HCl})$ 2-propanol. Cells were pelleted by centrifugation $(1000 \mathrm{~g}, 5 \mathrm{~min})$ and the absorbance of the supernatant was measured. For all assays, reactions containing no cells were used to determine blank values, which were subtracted from values obtained in assays with cells. 
Absorbance values of products formed were normalized with optical measurements of culture turbidity. Greater than $99.9 \%$ of cells were in the yeast form.

The ferric reductase assays rely on the formation of a red bathophenanthroline disulfonate (BPS)-Fe(II) complex, which is quantified by measuring absorbance at $515 \mathrm{~nm}$ (Dancis et al., 1990; Lesuisse et al., 1987). The source of ferric iron was $1 \mathrm{mM}$ ammonium ferric sulfate (Fluka) and the concentration of BPS (Sigma) was $1 \mathrm{mM}$.

The XTT reductase assay relies on the reduction of XTT to form a red formazan product, which is quantified by measuring absorbance at $515 \mathrm{~nm}$. XTT (Sigma) was prepared in standard reductase buffer and used at a concentration of $0.37 \mathrm{mM}$ with $0.5 \mu \mathrm{M}$ freshly made menadione (Sigma). Menadione was excluded from the XTT assay when superoxide dismutase (SOD) was included, as menadione can spontaneously yield superoxide following enzymic reduction (Hassan \& Fridovich, 1979). The absence of menadione decreased overall XTT activity by $\sim 20 \%$.

The WST-1 assays rely on reduction of WST-1 to form a yellow water-soluble formazan product, which is quantified by measuring absorbance at $450 \mathrm{~nm}$ (Ishiyama et al., 1993). WST-1 (Dojindo) was prepared as a $10 \mathrm{mM}$ stock in Milli-Q water and stored as aliquots at $-20^{\circ} \mathrm{C}$. Prior to use, an aliquot was thawed, diluted to $1 \mathrm{mM}$ in standard reductase buffer, and added to cells at a final concentration of $0.5 \mathrm{mM}$. Initial experiments demonstrated that the electron/hydrogen carrier 1-methoxy-5-methylphenazinium methylsulfate (1-mPMS; Dojindo), at $10 \mu \mathrm{M}$, had no effect (Student's $t$ test, $P=0 \cdot 845$ ) on the reduction of WST-1 (data not shown). This recapitulates the lack of effect of 1-mPMS on the reduction of WST-1 by activated neutrophils (Tan \& Berridge, 2000). Therefore, 1-mPMS was not included in the WST-1 assays.

The cellular reduction of MTT leads to the formation of insoluble indigo coloured crystals. MTT (Sigma) was prepared as a $1 \mathrm{~g} \mathrm{l}^{-1}$ stock solution in reductase buffer and combined with cells at a final concentration of $0.5 \mathrm{~g} \mathrm{l}^{-1}$. At the end of the assay, the formazan crystals were dissolved in acidified $(0.04 \mathrm{M} \mathrm{HCl})$ 2-propanol and the absorbance of the supernatant measured at $595 \mathrm{~nm}$.

In the reactions with iron-containing SOD (Sigma) from Escherichia coli, SOD was dissolved in $20 \mathrm{mM}$ HEPES and added at a $1: 10$ dilution to the suspended cells, immediately prior to addition of reductase substrate. Addition of HEPES buffer alone was used as control.

Measurement of oxygen consumption. The rate of cellular oxygen consumption was measured using a Clark-style electrode and a temperature-controlled chamber at $30{ }^{\circ} \mathrm{C}$ (System 203; Instech). The analogue signal was converted to a digital signal for data collection and analysis (Powerlab $2 / 25$ and software; ADInstruments). One million cells in exponential phase were diluted into prewarmed fresh media and placed into the sealed chamber. After a $30 \mathrm{~s}$ equilibration, recording was started and continued until dissolved oxygen was completely consumed. Oxygen consumption was linear with respect to time during this period.

\section{RESULTS}

\section{Ferric reductase and $\mathrm{XTT}$ activities are greater in $C$. albicans than in $S$. cerevisiae}

$\mathrm{NAD}(\mathrm{P}) \mathrm{H}$-dependent cell surface reductases are required for iron uptake, the ferrous product entering the cell through the Ftr/Fet-dependent high-affinity system. We previously observed that the rate of ferric iron uptake was 40-100-fold higher in C. albicans than in S. cerevisiae, and that at least $75 \%$ of this uptake was dependent on CaFRE10 for cells grown in low-iron, unbuffered defined medium (Knight et al., 2005). Direct comparison of whole-cell ferric reductase activity of $C$. albicans (wild-type strain SC5314) to a well-characterized S. cerevisiae strain (YPH499) after growth on low-iron defined media, indicated that the cell surface reductase activity of C. albicans was at least 10 -fold higher than that of $S$. cerevisiae $[12 \cdot 2 \pm 2 \cdot 3$ compared to $1 \cdot 1 \pm 0 \cdot 3 \mathrm{nmol} \mathrm{Fe}$ (II) $\left(10^{6} \text { cells }\right)^{-1} \mathrm{~h}^{-1}$, respectively, $n=5$ ]. Likewise XTT reduction was also considerably greater in C. albicans than in S. cerevisiae $[0 \cdot 117 \pm 0 \cdot 019$ compared to $0 \cdot 003 \pm 0.001\left(A_{515}\right)^{-1}\left(A_{720}\right)^{-1} \mathrm{~min}^{-1}$, respectively; values are means \pm SD for $n=5$ ].

\section{C. albicans XTT surface reductase activities: roles for CaFRE10, iron and $\mathrm{pH}$ regulation}

The activity of $C$. albicans responsible for the reduction of XTT to the formazan product was regulated by the level of iron in the growth medium prior to the assay (Fig. 1). This iron-regulated activity was reminiscent of the iron-regulated reduction of ferric compounds catalysed by CaFre10p (Knight et al., 2005). To determine if CaFre10p was involved

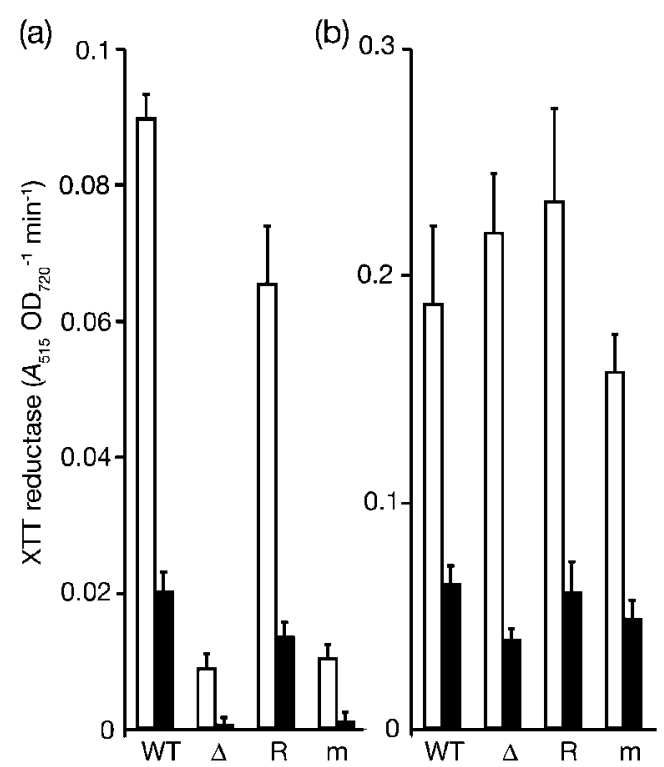

Fig. 1. C. albicans XTT reductase activity is regulated by iron and $\mathrm{pH}$, and is dependent on CaFRE1O in unbuffered medium. C. albicans strains SC5314 [wild-type (WT)], BCa15-2 $\left[\right.$ Cafre10 $\left.{ }^{-/-}(\Delta)\right]$, CT218.2 [Cafre10 ${ }^{-/-}+$CaFRE10 (R)], and CT212.2 [Cafre $10^{-1-}+$ Cafre10-1 $\left.(\mathrm{m})\right]$ were grown at $30^{\circ} \mathrm{C}$ in low-iron defined medium (open bars) or the same medium supplemented with $50 \mu \mathrm{M}$ ferric ammonium sulfate (solid bars). In exponential phase, the cells were washed and assayed for XTT reductase activity. (a) Results for cells grown in nonbuffered medium; (b) results for cells grown in the same medium buffered with $50 \mathrm{mM}$ HEPES to $\mathrm{pH} \mathrm{6.8.} \mathrm{Data} \mathrm{are}$ means $\pm S D$ for five independent cultures. 
in XTT reduction, a Cafre $10^{-/-}$homozygous deletion strain (strain BCa15-2; $\Delta$ ) was compared to SC5314 (WT) (Fig. 1a). When cells were grown in low-iron defined medium and then washed and assayed, wild-type C. albicans had 10-fold higher XTT activity than the Cafre10 ${ }^{-1-}$ mutant. C. albicans CT281.2, harbouring a reinserted functional CaFRE10 (R), restored XTT activity to $73 \%$ of that of SC5314, whereas reinsertion of a non-functional Cafre10-1 allele (strain CT212.2; m) had no effect. This genetic complementation of XTT activity was similar to that observed for ferric reductase activity (Knight et al., 2005). When grown in low-iron conditions, the Cafre $10^{-1-}$ mutant retained some XTT activity. However, when the Cafre10 ${ }^{-1-}$ mutant was grown in the presence of iron, the XTT activity was repressed to virtually zero (Fig. 1a). Overall, C. albicans XTT activity was regulated by the level of iron exposure during growth. Furthermore, for cells grown in unbuffered medium, in low or iron-supplemented conditions, the majority of the XTT activity was dependent on CaFRE10.

The transcript levels of genes CaFRE1 (orf19.8848), CaFRE2 (orf19.8849), CaFRE7 (orf19.6139) and CaFRE9 (orf19.3538), which have homology to CaFRE10, are upregulated at $\mathrm{pH} 8$ (Bensen et al., 2004). We had also previously observed that, for $C$. albicans grown at nearneutral $\mathrm{pH}, \mathrm{CaFRE} 10$ contributed very little to the total cell surface ferric reductase activity. In contrast, for cells grown in unbuffered ( $\mathrm{pH} 4 \cdot 0-4 \cdot 4)$ medium, CaFRE10 encodes the majority of the activity (Knight et al., 2005; and data not shown). To examine how the $\mathrm{pH}$ of the growth medium might affect the ability of $C$. albicans to reduce XTT, wildtype and CaFRE10 mutant strains were grown in defined medium with $50 \mathrm{mM}$ HEPES to maintain $\mathrm{pH}$ between $6 \cdot 7$ and $6 \cdot 8$ (Fig. 1b). At this near-neutral pH, XTT activity was independent of CaFRE10 (Fig. 1b), yet was still regulated by the amount of iron in the growth medium. In addition, the XTT activity of cells grown in buffered medium was two- to threefold greater than for cells grown in unbuffered medium (note different scale of Fig. 1a, b). The identity of the $C$. albicans gene(s) encoding the iron-regulated reductase activity expressed in buffered growth medium is unknown.

\section{Partial inhibition of WST-1 and XTT reduction by SOD}

WST-1 is a water-soluble tetrazolium salt that is membraneimpermeable and is reduced to a water-soluble formazan. It can be reduced by superoxide anion, and inhibition of this reaction by SOD can be used to assess superoxide production (Tan \& Berridge, 2000). Frequently, with assays that involve reduction of WST-1, an intermediate electron/ hydrogen carrier is employed. For WST-1, the electron/ hydrogen carrier is 1-mPMS. We observed no effect on the ability of C. albicans to reduce WST-1 in the presence or absence of 1-mPMS (data not shown), and therefore did not include 1-mPMS in the assays reported here. To investigate if superoxide was involved in WST- 1 and XTT reduction by C. albicans, SOD was added to the reactions immediately prior to the addition of WST-1 or XTT (Fig. 2a). In the presence of $30 \mu \mathrm{g} \mathrm{SOD} \mathrm{ml}{ }^{-1}$, WST-1 reductase activity was decreased to $74 \%$ of control values (no SOD). This suggests that the majority of WST-1 reduction was not mediated by superoxide. The presence of SOD $\left(30 \mu \mathrm{g} \mathrm{ml}^{-1}\right)$ had a greater inhibitory effect on XTT reduction, but most XTT reduction (64\% of control activity) was SOD-independent.

The standard assay buffer [50 mM citrate, $\mathrm{pH} 6 \cdot 6,5 \%(\mathrm{w} / \mathrm{v})$ D-glucose] that we used to assess WST-1 and XTT reductase activities is identical to the buffer used for the genetic and biochemical characterization of ScFRE1 in S. cerevisiae (Dancis et al., 1990; Lesuisse et al., 1987), and is based on earlier assays used to measure ferricyanide reduction (Avron \& Shavit, 1963). Citrate can function as a chemical reductant
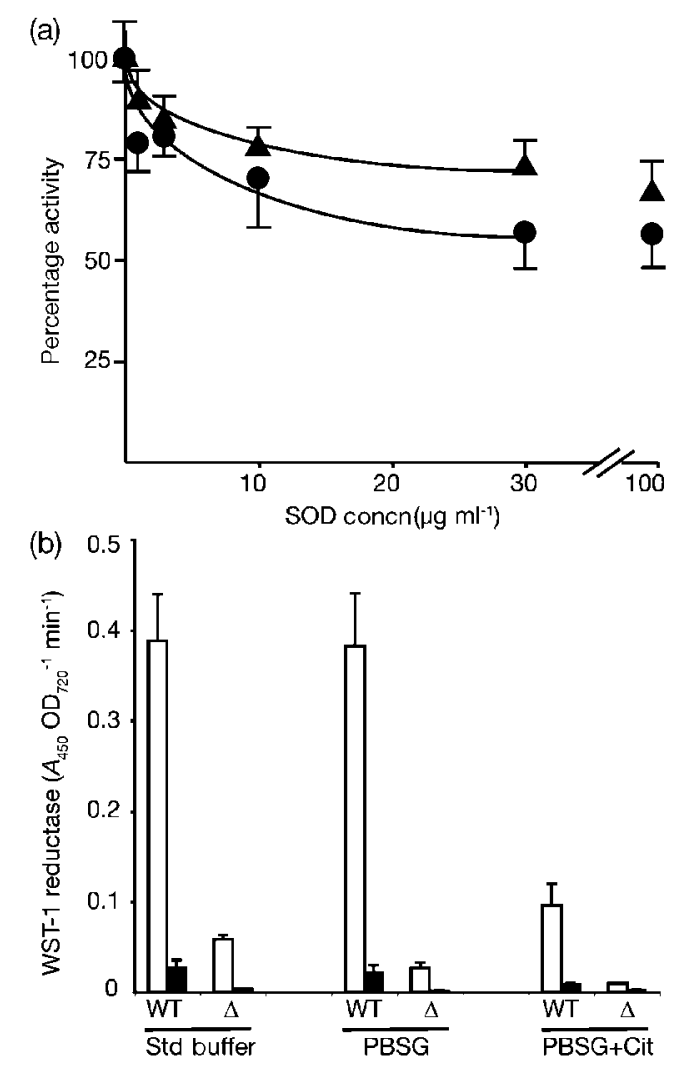

Fig. 2. C. albicans reduction of soluble tetrazolium salts is partially inhibited by SOD and independent of citrate. (a) Wildtype C. albicans was grown in low-iron defined media at $30^{\circ} \mathrm{C}$ to exponential phase. The cells were washed and assayed for XTT reductase activity (no menadione) $(\bullet)$ and WST-1 reductase activity $(\boldsymbol{\Delta})$ in the presence of SOD. (b) Wild-type (WT) and Cafre $10^{-1-}(\Delta)$ C. albicans were grown in low-iron defined medium (open bars) and the same medium supplemented with $50 \mu \mathrm{M}$ ferric ammonium sulfate (solid bars) at $30^{\circ} \mathrm{C}$. At exponential phase, the cells were washed in assay buffers of: $50 \mathrm{mM}$ citrate buffer, $\mathrm{pH} 6 \cdot 6$, with $5 \%(\mathrm{w} / \mathrm{v})$ D-glucose (Std buffer); Dulbecco's PBS, pH 7.2, with $5 \%$ D-glucose (PBSG); and PBSG with $50 \mathrm{mM}$ citrate, $\mathrm{pH} 7 \cdot 3$ (PBSG + Cit). The cells were assayed for WST-1 reductase activity in the corresponding buffers. Data are means \pm SD for five independent cultures. 
and is required for optimal ferric reductase activity of intact yeast cells. To test if citrate might be required to mediate reduction of WST-1, we compared the ability of wild-type and Cafre10 ${ }^{-1-}$ C. albicans to reduce WST-1 in our standard assay buffer and in Dulbecco's PBS, pH 7·2, supplemented with $5 \%(\mathrm{w} / \mathrm{v})$ D-glucose (PBSG) (Fig. 2b). As observed previously (Fig. 1a), there were clear effects of the presence of iron in the growth medium in down-regulating WST-1 activity, and the majority of the activity was CaFRE10dependent under both assay conditions. However, there was no difference in the rate of WST-1 reduction between cells assayed in standard assay buffer compared to those assayed in PBSG. Addition of $50 \mathrm{mM}$ citrate to PBSG shifted the $\mathrm{pH}$ to $7 \cdot 3$ and decreased the rate of WST- 1 reduction by threeto fourfold (Fig. 2b). The reason for this decrease is not known. Taken together, these results suggest that citrate does not mediate the cellular reduction of WST-1.

\section{C. albicans MTT intracellular reductase activities: iron regulation but no role for CaFRE10}

MTT has greater lipophilicity than XTT and WST-1, and is likely reduced by intracellular reductants (Berridge et al., 2005). Examination of MTT reduction with whole cells revealed little to no difference in MTT reductase activity between wild-type (Fig. 3a, W) and the Cafre10 $0^{-1-}$ mutant (Fig. 3a, $\Delta$ ), indicating that the reduction of MTT was not dependent on CaFre10p. Light microscopy of cells indicated that the reduced MTT product was associated with intracellular membrane structures, following the reductase assay (data not shown). Despite the lack of dependence on CaFRE10, MTT reduction was still iron-regulated. Three independently isolated C. albicans strains, SC5314 (W), WO-1 [White (Wh) and Opaque (Op) morphologies], and CBS5736 (W2), showed 5-10-fold more MTT reductase activity in iron-deprived compared with iron-replete cells. Both the MTT and the WST-1 reductase activities of the high-frequency switching strain WO-1 were less than those of strains SC5314 and CBS5736. Strain WO-1 was examined further after confirming the morphology of subtypes by microscopic examination of 200-500 cells from representative cultures. Under these mild conditions of iron limitation, there was little effect of iron on the switching process. The opaque cells had slightly, but consistently, higher MTT and WST-1 reductase activities.

MTT reductase activity in S. cerevisiae (Fig. 3a, Sc) was considerably less than that in C. albicans, and, like $C$. albicans, MTT reduction in S. cerevisiae was independent of the externally directed ferric reductases, encoded by ScFRE1 and ScFRE2 (data not shown). In contrast to C. albicans, there was no evidence that the low amount of MTT reduction observed in S. cerevisiae was iron-regulated. Deletion of mtDNA in $S$. cerevisiae $\left(\rho^{\circ}\right)$ caused a small but statistically significant (Student's $t$ test, $P=0.0002$ ) increase in the rate of MTT reduction, which was diminished when the cells were grown in the presence of iron (Fig. 3a). WST-1 reduction rates observed in $S$. cerevisiae were unaffected by

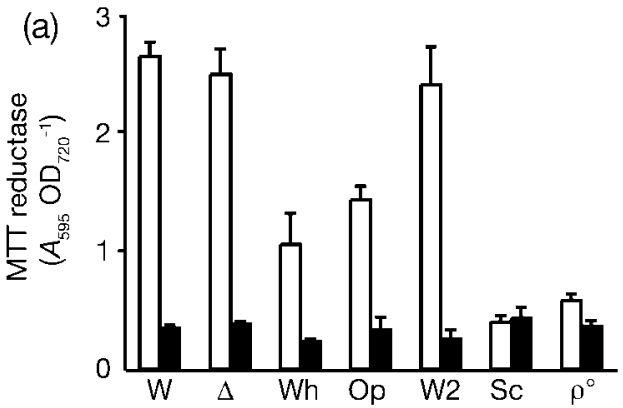

(b) 0.15

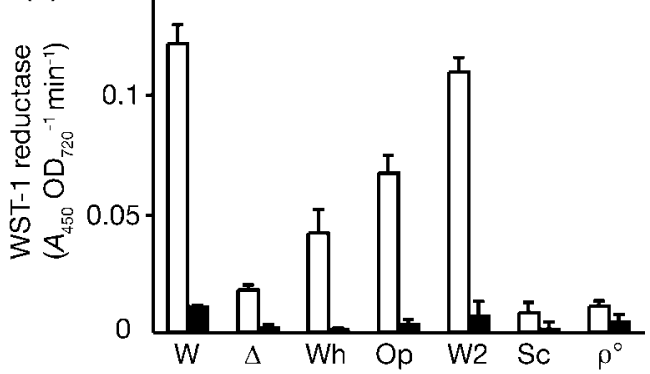

Fig. 3. MTT reductase activity is iron-regulated but independent of CaFRE10. C. albicans strains SC5314 [wild-type (W)], BCa15-2 [Cafre 10 ${ }^{-1-}(\Delta)$ ], WO-1 [white phase (Wh)], WO-1 [opaque phase (Op)], CBS5736 [wild-type (W2)] and S. cerevisiae strains $\mathrm{YPH} 499$ (Sc) and YPH250 $\rho^{\circ}\left(\rho^{\circ}\right)$ were grown in low-iron defined medium (open bars) and the same medium supplemented with $50 \mu \mathrm{M}$ ferric ammonium sulfate (solid bars) at $30^{\circ} \mathrm{C}$. At exponential phase, the cells were washed and assayed for MTT (a) and WST-1 (b) reductase activities. Data are means \pm SD for five independent cultures.

mtDNA status; however, the low values may preclude firm conclusions from being made (Fig. $3 \mathrm{~b}$ ). When we measured respiration directly in C. albicans, we did not observe any difference in the rate of oxygen consumption between the wild-type and the $\mathrm{Cafrel0}^{--1-}$ mutant strains (data not shown). These data suggest that the reductase activity encoded by CaFRE10 is independent of mitochondrial respiration.

\section{DISCUSSION}

Tetrazolium salt reductase assays have been used to assess the effectiveness of antifungal drugs by providing rapid, real-time and quantitative assay measurements of celldependent activities. The question addressed in this paper is, what enzyme(s) is responsible for XTT reduction when used to assess fungal and, in particular, C. albicans viability/ biomass? Using a genetic approach, we have demonstrated that the cell surface ferric reductase activity of $C$. albicans, encoded by CaFRE10, was primarily responsible for the reduction of XTT and WST-1 when C. albicans was grown in unbuffered medium.

The ability of $C$. albicans CaFre10p to reduce such structurally different substrates raises the possibility that an 
intermediate electron carrier performs reduction of ferric iron and tetrazolium salts. The standard XTT assay utilizes menadione, and protocols using WST-1 include 1-mPMS (Meletiadis et al., 2001; Scarlett et al., 2005). In our assays, omission of these two electron carrier intermediates from their respective assays led to only small decreases in tetrazolium reduction rates (20\% or less). Citrate is a major component of the reductase assay buffer (Avron \& Shavit, 1963; Dancis et al., 1990; Lesuisse et al., 1987); however, it was not required for reduction of WST-1. The presence of SOD did decrease the rate of tetrazolium reduction. However, most XTT and WST-1 reductase activity was SOD-independent. C. albicans is flavinogenic, and flavin secretion is increased when C. albicans is grown under ironlimiting conditions (Burkholder, 1943; Knight et al., 2002). In our study, the cells were washed before the enzyme assays; however, it cannot be excluded that sufficient flavin was secreted by $C$. albicans, during the $10-15 \mathrm{~min}$ assay, to mediate electron transfer from the reductase enzyme to the tetrazolium salt. The possibility that the ferric reductases can transfer electrons directly to the tetrazolium salts remains to be tested.

The expression of CaFRE10 is highly regulated by environmental conditions. Under iron-limiting conditions, CaFRE10 mRNA levels are substantially elevated (Knight et al., 2002). The increase in CaFRE10 expression under iron-limiting conditions is reflected in an increase in cell surface ferric reductase activity and, as shown in this study, an increase in the rate of reduction of XTT and WST-1. However, iron is not the only environmental factor that influences cell surface reductase activity. Changing the $\mathrm{pH}$ to near-neutral conditions increased cell surface reductase activity when ferric iron and XTT were electron acceptors, but the activity was independent of CaFRE10. Nonetheless, at near-neutral $\mathrm{pH}$, cell surface reductase activity was still repressed by environmental iron. There are a number of FRE homologues in C. albicans. Microarray data demonstrate that CaFRE10 is down-regulated at alkaline $\mathrm{pH}$ and that the expression of four other CaFRE homologues [CaFRE1 (orf19.8848), CaFRE2 (orf19.8849), CaFRE7 (orf19.6139), CaFRE9 (orf19.3538)] are upregulated at alkaline $\mathrm{pH}$ (Bensen et al., 2004). Although the gene(s) responsible for encoding the cell surface reductase at neutral and alkaline $\mathrm{pH}$ still needs to be identified, the regulated expression of these genes encoding ferric reductase homologues may have strong effects on tetrazolium reduction activity.

The tetrazolium salt MTT differs from XTT and WST-1 in that it is cell-permeable and can cross the plasma membrane (Berridge et al., 2005). Here, we have shown that the reduction of MTT in C. albicans was independent of the cell surface reductase encoded by CaFRE10. This observation is in agreement with the intracellular site of MTT reduction demonstrated in the rat B12 neural cell line (Liu et al., 1997). However, MTT reductase activity in C. albicans was iron-regulated. As stated above, there are a number of FRE homologues in C. albicans of unknown cellular location, and it is possible that one or more of these is responsible for the intracellular reduction of MTT. MTT reductase of $C$. albicans was much more active than that of S. cerevisiae, and only the C. albicans MTT reductase was iron-regulated. Perhaps C. albicans possesses a distinct regulatory circuit that links iron to $\mathrm{NAD}(\mathrm{P}) \mathrm{H}$ levels. The high reductase activities of $C$. albicans may distinguish it from nonpathogenic S. cerevisiae. Finally, the use of tetrazolium salts as indicators of $C$. albicans viability/biomass must be re-evaluated in view of the fact that the enzymes that catalyse the reduction of these compounds are highly regulated by growth conditions.

\section{ACKNOWLEDGEMENTS}

This work was supported by grants NIH (RO1 AI052384) to A. D. and $\mathrm{NIH}$ (RO3 AI063437) to S.K. Sequence data for C. albicans was obtained from the Stanford Genome Technology Center website at http://www-sequence.stanford.edu/group/candida. Sequencing of $C$. albicans was accomplished with the support of the National Institute of Dental and Craniofacial Research (NIDCR) and the Burroughs Wellcome Fund. We are very grateful to Burk Braun, Alexander Johnson and David Soll for C. albicans strains.

\section{REFERENCES}

Arnaud, M. B., Costanzo, M. C., Skrzypek, M. S., Binkley, G., Lane, C., Miyasato, S. R. \& Sherlock, G. (2005). The Candida Genome Database (CGD), a community resource for Candida albicans gene and protein information. Nucleic Acids Res 33, D358-D363.

Askwith, C., Eide, D., Van Ho, A., Bernard, P. S., Li, L., Davis-Kaplan, S., Sipe, D. M. \& Kaplan, J. (1994). The FET3 gene of S. cerevisiae encodes a multicopper oxidase required for ferrous iron uptake. Cell 76, 403-410.

Avron, M. \& Shavit, N. (1963). A sensitive and simple method for determination of ferrocyanide. Anal Biochem 6, 549-554.

Bensen, E. S., Martin, S. J., Li, M., Berman, J. \& Davis, D. A. (2004). Transcriptional profiling in Candida albicans reveals new adaptive responses to extracellular $\mathrm{pH}$ and functions for Rim101p. Mol Microbiol 54, 1335-1351.

Berridge, M. V. \& Tan, A. S. (1993). Characterization of the cellular reduction of 3-(4,5-dimethylthiazol-2-yl)-2,5-diphenyltetrazolium bromide (MTT): subcellular localization, substrate dependence, and involvement of mitochondrial electron transport in MTT reduction. Arch Biochem Biophys 303, 474-482.

Berridge, M. V. \& Tan, A. S. (2000). Cell-surface NAD(P)H-oxidase: relationship to trans-plasma membrane $\mathrm{NADH}$-oxidoreductase and a potential source of circulating NADH-oxidase. Antioxid Redox Signal 2, 277-288.

Berridge, M. V., Herst, P. M. \& Tan, A. S. (2005). Tetrazolium dyes as tools in cell biology: new insights into their cellular reduction. Biotechnol Annu Rev 11, 127-152.

Blaiseau, P. L., Lesuisse, E. \& Camadro, J. M. (2001). Aft2p, a novel iron-regulated transcription activator that modulates, with Aft $1 \mathrm{p}$, intracellular iron use and resistance to oxidative stress in yeast. J Biol Chem 276, 34221-34226.

Braun, B. R., Head, W. S., Wang, M. X. \& Johnson, A. D. (2000). Identification and characterization of TUP1-regulated genes in Candida albicans. Genetics 156, 31-44. 
Burkholder, P. R. (1943). Synthesis of riboflavin by a yeast. Proc Natl Acad Sci U S A 29, 166-172.

Courel, M., Lallet, S., Camadro, J. M. \& Blaiseau, P. L. (2005). Direct activation of genes involved in intracellular iron use by the yeast iron-responsive transcription factor Aft2 without its paralog Aft1. Mol Cell Biol 25, 6760-6771.

Crane, F. L., Roberts, H., Linnane, A. W. \& Low, H. (1982). Transmembrane ferricyanide reduction by cells of the yeast Saccharomyces cerevisiae. J Bioenerg Biomembr 14, 191-205.

Crane, F. L., Sun, I. L., Clark, M. G., Grebing, C. \& Low, H. (1985). Transplasma-membrane redox systems in growth and development. Biochim Biophys Acta 811, 233-264.

Dancis, A., Klausner, R. D., Hinnebusch, A. G. \& Barriocanal, J. G. (1990). Genetic evidence that ferric reductase is required for iron uptake in Saccharomyces cerevisiae. Mol Cell Biol 10, 2294-2301.

Dancis, A., Roman, D. G., Anderson, G. J., Hinnebusch, A. G. \& Klausner, R. D. (1992). Ferric reductase of Saccharomyces cerevisiae: molecular characterization, role in iron uptake, and transcriptional control by iron. Proc Natl Acad Sci U S A 89, 3869-3873.

Eck, R., Hundt, S., Hartl, A., Roemer, E. \& Kunkel, W. (1999). A multicopper oxidase gene from Candida albicans: cloning, characterization and disruption. Microbiology 145, 2415-2422.

Eide, D., Davis-Kaplan, S., Jordan, I., Sipe, D. \& Kaplan, J. (1992). Regulation of iron uptake in Saccharomyces cerevisiae. The ferrireductase and $\mathrm{Fe}(\mathrm{II})$ transporter are regulated independently. J Biol Chem 267, 20774-20781.

Finegold, A. A., Shatwell, K. P., Segal, A. W., Klausner, R. D. \& Dancis, A. (1996). Intramembrane bis-heme motif for transmembrane electron transport conserved in a yeast iron reductase and the human NADPH oxidase. J Biol Chem 271, 31021-31024.

Fox, T. D., Folley, L. S., Mulero, J. J. W. M. T., Thorness, P. E., Hedin, L. O. \& Costanzo, M. C. (1991). Analysis and manipulation of yeast mitochondrial genes. Methods Enzymol 194, 149-165.

Georgatsou, E. \& Alexandraki, D. (1994). Two distinctly regulated genes are required for ferric reduction, the first step of iron uptake in Saccharomyces cerevisiae. Mol Cell Biol 14, 3065-3073.

Hassan, H. M. \& Fridovich, I. (1979). Intracellular production of superoxide radical and of hydrogen peroxide by redox active compounds. Arch Biochem Biophys 196, 385-395.

Hassett, R. \& Kosman, D. J. (1995). Evidence for $\mathrm{Cu(II)} \mathrm{reduction} \mathrm{as}$ a component of copper uptake by Saccharomyces cerevisiae. J Biol Chem 270, 128-134.

Heyn, K., Tredup, A., Salvenmoser, S. \& Muller, F. M. (2005). Effect of voriconazole combined with micafungin against Candida, Aspergillus, and Scedosporium spp. and Fusarium solani. Antimicrob Agents Chemother 49, 5157-5159.

Ishiyama, M., Shiga, M., Sasamoto, K., Mizoguchi, M. \& He, P. (1993). A new sulfonated tetrazolium salt that produces a highly water-soluble formazan dye. Chem Pharm Bull 41, 1118-1122.

Johnsen, A. R., Bendixen, K. \& Karlson, U. (2002). Detection of microbial growth on polycyclic aromatic hydrocarbons in microtiter plates by using the respiration indicator WST-1. Appl Environ Microbiol 68, 2683-2689.

Kim, C., Crane, F. L., Faulk, W. P. \& Morre, D. J. (2002). Purification and characterization of a doxorubicin-inhibited NADH-quinone (NADH-ferricyanide) reductase from rat liver plasma membranes. J Biol Chem 277, 16441-16447.

Knight, S. A. B., Lesuisse, E., Stearman, R., Klausner, R. D. \& Dancis, A. (2002). Reductive iron uptake by Candida albicans: role of copper, iron and the TUP1 regulator. Microbiology 148, $29-40$.
Knight, S. A., Vilaire, G., Lesuisse, E. \& Dancis, A. (2005). Iron acquisition from transferrin by Candida albicans depends on the reductive pathway. Infect Immun 73, 5482-5492.

Kosman, D. J. (2003). Molecular mechanisms of iron uptake in fungi. Mol Microbiol 47, 1185-1197.

Kuhn, D. M., Balkis, M., Chandra, J., Mukherjee, P. K. \& Ghannoum, M. A. (2003). Uses and limitations of the XTT assay in studies of Candida growth and metabolism. J Clin Microbiol 41, 506-508.

Lan, C. Y., Rodarte, G., Murillo, L. A., Jones, T., Davis, R. W., Dungan, J., Newport, G. \& Agabian, N. (2004). Regulatory networks affected by iron availability in Candida albicans. Mol Microbiol 53, 1451-1469.

Lara-Ortiz, T., Riveros-Rosas, H. \& Aguirre, J. (2003). Reactive oxygen species generated by microbial NADPH oxidase NoxA regulate sexual development in Aspergillus nidulans. Mol Microbiol 50, 1241-1255.

Lesuisse, E. \& Labbe, P. (1994). Reductive iron assimilation in Saccharomyces cerevisiae. In Metal Ions in Fungi, pp. 149-178. Edited by G. Winkelmann \& D. R. Winge. New York: Marcel Dekker.

Lesuisse, E., Raguzzi, F. \& Crichton, R. R. (1987). Iron uptake by the yeast Saccharomyces cerevisiae: involvement of a reduction step. J Gen Microbiol 133, 3229-3236.

Liu, Y., Peterson, D. A., Kimura, H. \& Schubert, D. (1997). Mechanism of cellular 3-(4,5-dimethylthiazol-2-yl)-2,5-diphenyltetrazolium bromide (MTT) reduction. J Neurochem 69, 581-593.

McCluskey, C., Quinn, J. P. \& McGrath, J. W. (2005). An evaluation of three new-generation tetrazolium salts for the measurement of respiratory activity in activated sludge microorganisms. Microb Ecol 49, 379-387.

Meletiadis, J., Mouton, J. W., Meis, J. F., Bouman, B. A., Donnelly, J. P., Verweij, P. E. \& Network, E. (2001). Colorimetric assay for antifungal susceptibility testing of Aspergillus species. J Clin Microbiol 39, 3402-3408.

Morre, D. J. (2004). Quinone oxidoreductases of the plasma membrane. Methods Enzymol 378, 179-199.

Mosmann, T. (1983). Rapid colorimetric assay for cellular growth and survival: application to proliferation and cytotoxicity assays. J Immunol Methods 65, 55-63.

Paull, K. D., Shoemaker, R. H., Boyd, M. R. \& 10 other authors (1988). The synthesis of XTT: $a$ new tetrazolium reagent that is bioreducible to a water-soluble formazan. J Heterocycl Chem 25, 911-914.

Peskin, A. V. \& Winterbourn, C. C. (2000). A microtiter plate assay for superoxide dismutase using a water-soluble tetrazolium salt (WST-1). Clin Chim Acta 293, 157-166.

Ramalho, P. A., Paiva, S., Cavaco-Paulo, A., Casal, M., Cardoso, M. H. \& Ramalho, M. T. (2005). Azo reductase activity of intact Saccharomyces cerevisiae cells is dependent on the Frelp component of plasma membrane ferric reductase. Appl Environ Microbiol 71, 3882-3888.

Ramanan, N. \& Wang, Y. (2000). A high-affinity iron permease essential for Candida albicans virulence. Science 288, 1062-1064.

Roehm, N. W., Rodgers, G. H., Hatfield, S. M. \& Glasebrook, A. L. (1991). An improved colorimetric assay for cell proliferation and viability utilizing the tetrazolium salt XTT. J Immunol Methods 142, 257-265.

Rutherford, J. C., Ojeda, L., Balk, J., Muhlenhoff, U., Lill, R. \& Winge, D. R. (2005). Activation of the iron regulon by the yeast Aft $1 / \mathrm{Aft} 2$ transcription factors depends on mitochondrial but not cytosolic iron-sulfur protein biogenesis. J Biol Chem 280, 10135-10140.

Scarlett, D. J., Herst, P. M. \& Berridge, M. V. (2005). Multiple proteins with single activities or a single protein with multiple 
activities: the conundrum of cell surface NADH oxidoreductases. Biochim Biophys Acta 1708, 108-119.

Scudiero, D. A., Shoemaker, R. H., Paull, K. D., Monks, A., Tierney, S., Nofziger, T. H., Currens, M. J., Seniff, D. \& Boyd, M. R. (1988). Evaluation of a soluble tetrazolium/formazan assay for cell growth and drug sensitivity in culture using human and other tumor cell lines. Cancer Res 48, 4827-4833.

Segal, A. W. (2005). How neutrophils kill microbes. Annu Rev Immunol 23, 197-223.

Sikorski, R. S. \& Boeke, J. D. (1991). In vitro mutagenesis and plasmid shuffling: from cloned gene to mutant yeast. Methods Enzymol 194, 302-318.

Slutsky, B., Staebell, M., Anderson, J., Risen, L., Pfaller, M. \& Soll, D. R. (1987). "White-opaque transition": a second high-frequency switching system in Candida albicans. J Bacteriol 169, 189-197.

Stearman, R., Yuan, D. S., Yamaguchi-Iwai, Y., Klausner, R. D. \& Dancis, A. (1996). A permease-oxidase complex involved in highaffinity iron uptake in yeast. Science 271, 1552-1557.

Tan, A. S. \& Berridge, M. V. (2000). Superoxide produced by activated neutrophils efficiently reduces the tetrazolium salt, WST-1 to produce a soluble formazan: a simple colorimetric assay for measuring respiratory burst activation and for screening anti-inflammatory agents. J Immunol Methods 238, 59-68.
Tellier, R., Krajden, M., Grigoriew, G. A. \& Campbell, I. (1992). Innovative endpoint determination system for antifungal susceptibility testing of yeasts. Antimicrob Agents Chemother 36, 1619-1625.

Vignais, P. V. (2002). The superoxide-generating NADPH oxidase: structural aspects and activation mechanism. Cell Mol Life Sci 59, $1428-1459$.

Villalba, J. M., Navarro, F., Cordoba, F., Serrano, A., Arroyo, A., Crane, F. L. \& Navas, P. (1995). Coenzyme Q reductase from liver plasma membrane: purification and role in trans-plasma-membrane electron transport. Proc Natl Acad Sci U S A 92, 4887-4891.

Vistica, D. T., Skehan, P., Scudiero, D., Monks, A., Pittman, A. \& Boyd, M. R. (1991). Tetrazolium-based assays for cellular viability: a critical examination of selected parameters affecting formazan production. [erratum appears in Cancer Res 1991 Aug 15; 51(16):4501]. Cancer Res 51, 2515-2520.

Wiederhold, N. P., Kontoyiannis, D. P., Prince, R. A. \& Lewis, R. E. (2005). Attenuation of the activity of caspofungin at high concentrations against Candida albicans: possible role of cell wall integrity and calcineurin pathways. Antimicrob Agents Chemother 49, 5146-5148.

Yamaguchi-Iwai, Y., Stearman, R., Dancis, A. \& Klausner, R. D. (1996). Iron-regulated DNA binding by the AFT1 protein controls the iron regulon in yeast. $E M B O J 15,3377-3384$. 